\title{
Moving Pixel Displacement Detection using Correlation Functions on CIS Image
}

\author{
Kwang Ryol Ryu and Young Bin Kim, Member, KIMICS
}

\begin{abstract}
Moving pixel displacement detection algorithm using correlation functions for making panorama image on the continuous images is presented in this paper. The input images get from a CMOS image sensor (CIS). The camera is maintained by constant brightness and uniform sensing area in test input pattern. For simple navigation and capture image has to $70 \%$ overlapped region. A correlation rate in two image data is evaluated by using reference image with first captures, and compare image with next captures. The displacement of the two images are expressed to secondorder function of $x, y$ and solved with finding the coefficient in second order function. That results in the change in the peak correlation displacement from the reference to the compare image, is moving to pixel length. The navigating error is reduced by varying the path because the error is shown in the difference of the positioning vector between the true pixel position and the navigated pixel position. The algorithm performance is evaluated to be different from the error vector to vary the navigating path grid.
\end{abstract}

Index Terms- Moving pixel displacement, correlation function, CIS image, vector error, correlation navigator.

\section{INTRODUCTION}

The image stitching is required to navigate the coordinates to separated neighbor images to composite for sure at the freehand image scanning and floating image with Complementary Metal Oxide Semiconductor (CMOS) Image Sensor (CIS). CIS is traditionally more susceptible to noise, consumes low power, one chip processor and low cost to be a good embedded system for an image and video processing system. The several separated scanning images out of the one image need a positioning of the coordinate to seam for complete composition. A panorama image is made of compositing with a number of image shots. Two images $I_{1}$ and $I_{2}$ capture different portions of the same scene with an overlapping region viewed in both images.[1-4] The images should be stitched to generate a mosaic image $I$. A simple pasting of a left region from $I_{1}$ and a right region from $I_{2}$ produces visible artificial contours in the seaming between the images due to differences of camera

\footnotetext{
Manuscript received July 1, 2010; accepted July 1, 2010.

Kwang Ryol Ryu and Young Bin Kim are with Department of Electronic Engineering, Mokwon University, Daejeon, 302-729, S. Korea (Tel:+82-42829-7651, Email: conan@mwu.ac.kr)
}

gain and scene illumination or geometrical misalignments.[5,6] The purpose of a stitching algorithm is to produce a visually plausible mosaic with two desirable properties. That is required to apply various techniques like geometric transformation, multi-scale and blending. [7,8] Thus this paper proposes the task of finding points in overlapped images that these are aligned to accurate. A new algorithm with correlation function and navigating path grid are proposed for reducing the vector error of displacements. The method performance is evaluated in comparing with the proposed approach and alternative methods.

\section{PIXEL DISPLACEMENT DETECTIING ALGORITHM}

\section{A. Image Correlation}

The center position of the captured image using a spatial sensor is overlapped as shown in Fig. 1 not to be transparent. For the solving problem it is important to find a matching area between a current and previous image. The system subsequently performs real-time correlation processing using a nonzero-mean difference detection function. The real time correlation is described by the expression (1),

$$
C_{i, j}^{k}=\sum_{m=1}^{M} \sum_{n=1}^{N}\left|r_{m, n}-c_{m-i, n-j}\right|^{k}
$$

where $r_{m, n}\left(c_{m, n}\right)$ is the digitized value of the reference and comparison image at pixel $\{m, n\}$, and $i, j \in \mathrm{Z}$ represents the shift of the comparison frame which is relative to the reference frame measured in units of pixels. Typically $k \in N$ in principle satisfies $k \in R>1$. To get the relative image over the same geographic region of the test pattern, the elliptic correlation surface is given in the expression (1). The first step is modeling about accurate correlation function. The correlation surface is made of the sum-of difference-squared as shown in Fig. 2. The accurate description of correlation surface results from the making expression for the solving of system configuration. 


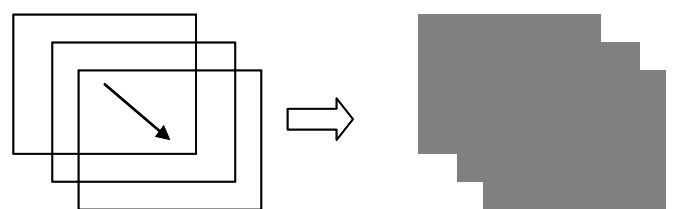

Fig. 1 Capture image series and image overlap

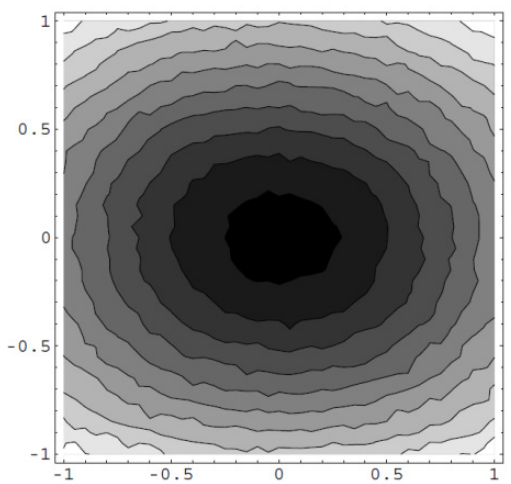

Fig. 2 Correlation surface contour

A second-order function of $\mathrm{x}$ and $\mathrm{y}$ is given in $z^{\prime}=f^{\prime}\left(x^{\prime}, y^{\prime}\right)$. It is considering a simple elliptic paraboloid, an initial idealized three-dimensional model of the system correlation surface is described by the expression (2).

$$
z^{\prime}=f^{\prime}\left(x^{\prime}, y^{\prime}\right)=q_{20} x^{\prime 2}+q_{02} y^{\prime 2}
$$

If $q_{20}, q_{02}>0$, then the surface has a unique minimum $z^{\prime}=0$ at $\left\{x^{\prime}, y^{\prime}\right\}=\{0,0\}$. In this case, the major and minor axes of the ellipse are formed by the intersection of $f^{\prime}\left(x^{\prime}, y^{\prime}\right)$ with a particular contour plane $z^{\prime}=z_{0} \equiv$ constant are given in $\sqrt{z_{0} / q_{20}}$ and $\sqrt{z_{0} / q_{02}}$, respectively. The $\left\{x^{\prime}, y^{\prime}\right\}$ plane is transformed to the surface $z^{\prime}=f^{\prime}\left(x^{\prime}, y^{\prime}\right)$ using twodimensional affine transformation, and the inverse of that transformation can be represented in the Cartesian basis by the matrix expression (3).

$$
c=\left[\begin{array}{ll}
c_{11} & c_{12} \\
c_{21} & c_{22}
\end{array}\right]
$$

In the case of a rotation about the $\mathrm{z}^{\prime}$ axis by an angle $\phi$, $c$ is described by the expression (4).

$$
c \equiv R(\phi)=\left[\begin{array}{cc}
\cos \phi & \sin \phi \\
-\sin \phi & \cos \phi
\end{array}\right]
$$

However, the expression (3) also is converted to 2D transformations such as shears and reflections as well as arbitrary compositions of subsequent rotations. Next, the application of the 2D affine transformation is converted to 3D translation by the vector $\left\{x_{0}, y_{0}, z_{0}\right\}$, and the laboratory reference frame is specified by the coordinates $\{x, y, z\}$. The original coordinates of the undisturbed correlation surface is produced in terms of the laboratory coordinates by inverting both the translation and the affine transformation in order as the equations (5) to (7),

$$
\begin{aligned}
& x^{\prime}=c_{11}\left(x-x_{0}\right)+c_{12}\left(y-y_{0}\right) \\
& y^{\prime}=c_{21}\left(x-x_{0}\right)+c_{22}\left(y-y_{0}\right) \\
& z^{\prime}=z-z_{0}
\end{aligned}
$$

The transformed model surface in the laboratory coordinate system is given in the functional expression $\{x, y, z\}$. By substituting equations (5)-(7) into (2), the result is $z=f(x, y)$,

$$
\begin{aligned}
f(x, y)= & a_{00}+a_{10} x+a_{01} y+a_{20} x^{2}+a_{11} x y+a_{02} y^{2} \\
a_{20} & =q_{20} c_{11}^{2}+q_{02} c_{21}^{2}, \\
a_{02} & =q_{20} c_{12}^{2}+q_{02} c_{22}^{2}, \\
a_{11} & =2\left(q_{20} c_{11}^{2} c_{12}+q_{02} c_{21} c_{22},\right. \\
a_{10} & =-\left(a_{11} y_{0}+2 a_{20} x_{0}\right), \\
a_{01} & =-\left(a_{11} x_{0}+2 a_{02} y_{0}\right), \\
a_{00} & \left.=z_{0}+a_{11} x_{0} y_{0}+a_{20} x_{0}^{2}+a_{02} y_{0}^{2}\right),
\end{aligned}
$$

The transformed surface in the laboratory coordinate system has six terms of the expression $a_{m n} x^{m} y^{n}$, where $\{m, n\} \in\{0,1,2\}$ for $0 \leq m+n \leq 2$. In addition the two coefficients $a_{10}$ and $a_{01}$ can be uniquely expressed in terms of the displacements $\left\{x_{0}, y_{0}\right\}$ as the expression (10) and (11).

$$
\begin{aligned}
& x_{0}=\frac{a_{01} a_{11}-2 a_{10} a_{02}}{4 a_{20} a_{02}-a_{11}^{2}} \\
& y_{0}=\frac{a_{10} a_{11}-2 a_{01} a_{20}}{4 a_{20} a_{02}-a_{11}^{2}}
\end{aligned}
$$

As a result, the value of transform vector, $\{\mathrm{x} 0, \mathrm{y} 0\}$ can be obtained to five coefficient, without find the two coefficient value of q2,0 and q0,2 or inverse transform of matrix $\mathrm{C}$. 


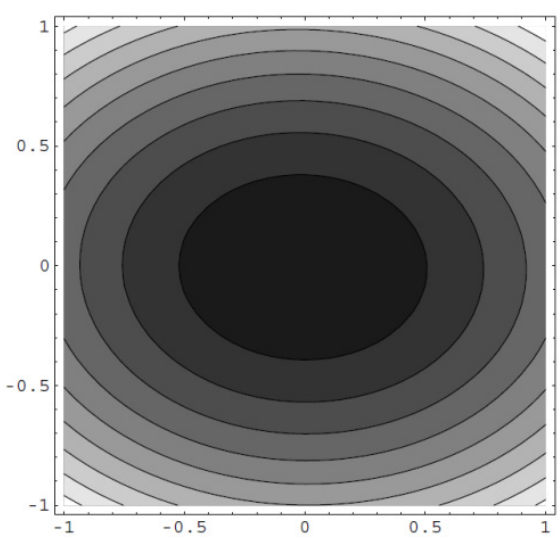

Fig. 3 Best least-squares fit surface

Fig. 3 shows the best numerical least-squares fit surface of (8) for the data of in Fig. 2 obtained by applying the model function.

\section{B. Linear Least-squares}

The correlation bowl is described by the correlation function $C_{i, j}^{k}$ in computing on the regular defined grid. For example, $i, j \in\{-1,0,1\}$ yields a square $3 \times 3$ grid containing nine correlation numbers with $C_{0,0}^{k}$ located in the center cell. Consider then the general problem of finding the best second-order model capable of representing this limited set of pixel shift correlation data by the navigator. That is, the case of the $3 \times 3$ correlation gird is required to find the value of the coefficients $\left\{a_{m n}\right\}$ that allow $f(x, y)$ to model the sum-ofsquared-differences correlation data as accurately as possible. The mapping between the $3 \times 3$ navigator pixel-shift correlation grid and the corresponding values of $f(x, y)$ as defined by (8) is shown in Fig. 4 schematically.

\begin{tabular}{|l|l|l|}
\hline$C_{2}$ & $C_{3}$ & $C_{4}$ \\
\hline$C_{1}$ & $C_{0}$ & $C_{5}$ \\
\hline$C_{8}$ & $C_{7}$ & $C_{6}$ \\
\hline
\end{tabular}$\longrightarrow$\begin{tabular}{|l|l|l|}
$f(-1,1)$ & $f(0,1)$ & $f(1,1)$ \\
\hline$f(-1,0)$ & $f(0,0)$ & $f(1,0)$ \\
\hline$f(-1,-1)$ & $f(0,-1)$ & $f(1,-1)$ \\
\hline
\end{tabular}

Fig. 4 Schematic correlation navigator $3 \times 3$

The sum of the squared deviations between the correlation values and the corresponding model are estimated and made by using $f(x, y)$,

$$
\begin{aligned}
x^{2}= & {\left[c_{0}-f(0,0)\right]^{2}+\left[c_{1}-f(-1,0)\right]^{2} } \\
& +\left[c_{2}-f(-1,1)\right]^{2}+\left[c_{3}-f(0,1)\right]^{2} \\
& +\left[c_{4}-f(1,1)\right]^{2}+\left[c_{5}-f(1,0)\right]^{2} \\
& +\left[c_{6}-f(1,-1)\right]^{2}+\left[c_{7}-f(0,-1)\right]^{2} \\
& +\left[c_{8}-f(-1,-1)\right]^{2}
\end{aligned}
$$

A numerical fit of $f(x, y)$ to the nine correlation values $\left\{\mathrm{C}_{0}, \ldots, \mathrm{C}_{8}\right\}$ is also not practical in a real-time system. To solve the problem, pursue a purely analytic solution instead. At the minimum of $x^{2}$, the gradient with respect to the members of the parameter set $\left\{a_{m n}\right\}$ must vanish identically.

$$
\frac{\partial}{\partial a_{m n}} x^{2}=0
$$

The six independent parameters can be obtained from unknown parameters. For the specific case of the $3 \times 3$ navigator grid, the parameter is given below:

$a_{00}=\frac{1}{9}\left[5 c_{0}+2\left(c_{1}+c_{3}+c_{5}-c_{7}\right)-\left(c_{2}+c_{4}+c_{6}+c_{8}\right)\right]$

$a_{10}=\frac{1}{6}\left[\left(c_{4}+c_{5}+c_{6}\right)-\left(c_{1}+c_{2}+c_{8}\right)\right]$

$a_{01}=\frac{1}{6}\left[\left(c_{2}+c_{3}+c_{4}\right)-\left(c_{6}+c_{7}+c_{8}\right)\right]$

$a_{11}=\frac{1}{4}\left[\left(c_{4}+c_{8}\right)-\left(c_{2}+c_{6}\right)\right]$

$a_{20}=\frac{1}{6}\left[\left(c_{1}+c_{2}+c_{8}+c_{4}+c_{5}+c_{6}\right)-2\left(c_{0}+c_{3}+c_{7}\right)\right]$

$a_{02}=\frac{1}{6}\left[\left(c_{2}+c_{3}+c_{4}+c_{6}+c_{7}+c_{8}\right)-2\left(c_{0}+c_{1}+c_{5}\right)\right]$

\section{Cell extension}

Consider a navigation sensor based on an optical system with a resolution that is significantly smaller than the mean correlation length of the paper fibers under test. If the system has sufficient processor power, it is possible to sample the true correlation surface on a larger gird than $3 \times 3$ with the intent of reducing the sensitivity of the navigation algorithm for statistical fluctuations in the correlation processing thereby increasing navigation accuracy. It can be choose $i, j \in\{-2,-1,0,1,2\}$ to yield square $5 \times 5$ grid containing twenty five correlation 
numbers with $C_{0,0}^{k}$ located in the center cell as shown schematically in Fig 5. As mentioned, the value for the coefficients $\left\{a_{m n}\right\}$ is required to find in applying $f(x, y)$ to model the correlation data as accurate as possible. Using the same analytic $x^{2}$ minimization procedure, the results obtain the expressions that depend on the values of the twenty five correlation samples taken from the grid path.

\begin{tabular}{|l|l|l|l|l|}
\hline$C_{12}$ & $C_{13}$ & $C_{14}$ & $C_{15}$ & $C_{16}$ \\
\hline$C_{11}$ & $C_{02}$ & $C_{03}$ & $C_{04}$ & $C_{17}$ \\
\hline$C_{10}$ & $C_{01}$ & $C_{00}$ & $C_{05}$ & $C_{18}$ \\
\hline$C_{09}$ & $C_{08}$ & $C_{07}$ & $C_{06}$ & $C_{19}$ \\
\hline$C_{24}$ & $C_{23}$ & $C_{22}$ & $C_{21}$ & $C_{20}$ \\
\hline
\end{tabular}

Fig. 5 Schematic correlation navigator $5 \times 5$

\section{Cubic Correlation Function}

The performance of accurate pixel movement find depends on difference of fit second-order surface and true correlation surface. A linear correlation function (given by (1) with $\mathrm{k}=1$ ) is shown in Fig. 6, a linear correlation function actually generates a surface which is more difficult to represent with a second-order approximation than that of the quadratic correlation function illustrated in Fig. 7. Based on these results, we can introduce that a cubic correlation function would generate smaller errors than those produced by a quadratic function. The correlation surface compares with true correlation as show in Fig. 8.

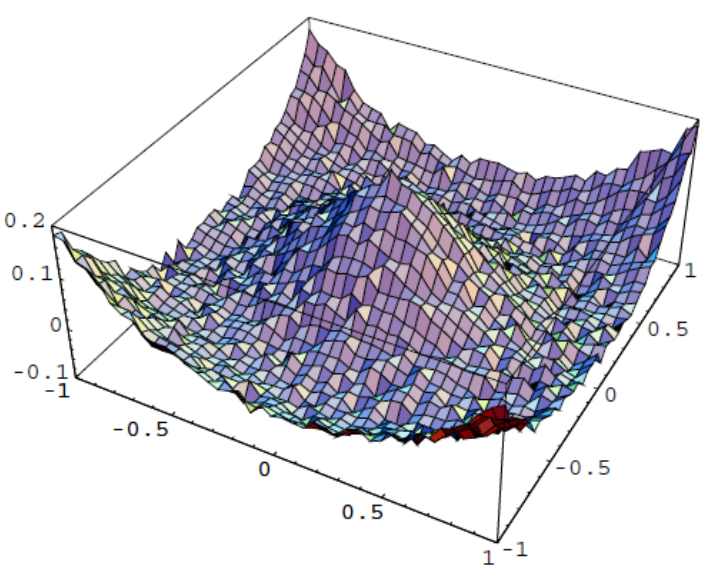

Fig. 6 Difference between the best fit second-order surface and the true correlation surface computed using a linear correlation function $\mathrm{k}=1$.

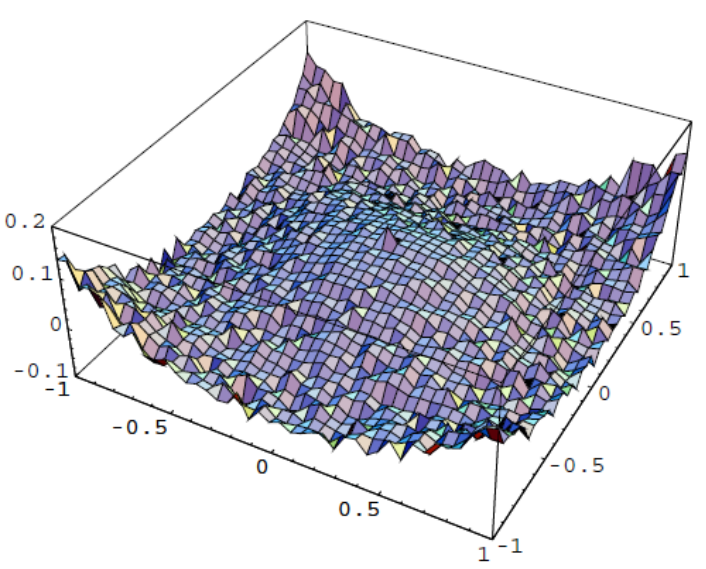

Fig. 7 Difference between the best fit second-order surface and the true correlation surface computed using a quadratic correlation function $\mathrm{k}=2$.

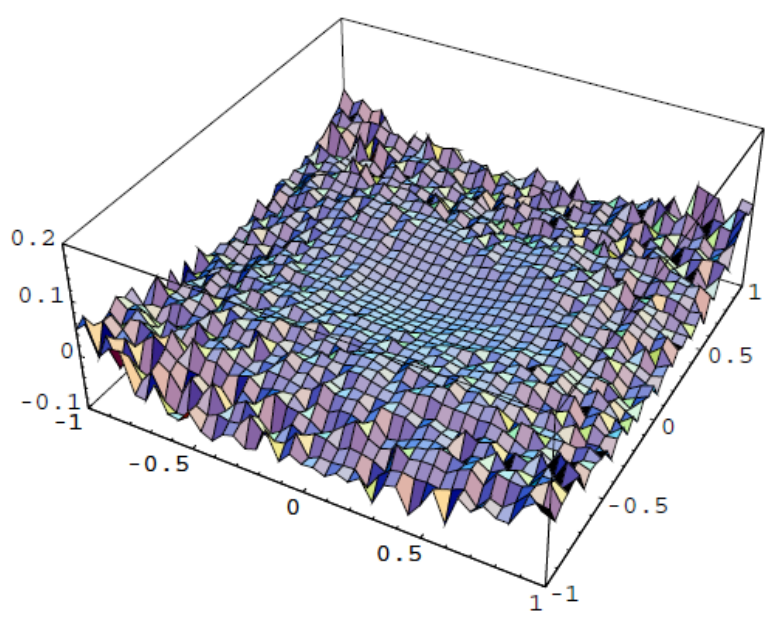

Fig. 8 Difference between the best fit second-order surface and the true correlation surface computed using a cubic correlation function $\mathrm{k}=3$.

\section{EXPERIMENT AND RESULTS}

The test image pattern showed in figure 9 is used to simulate for sensor input. The analysis leading to equation (9) (14) show that in the case of the elliptic paraboloid, the coefficients which determine the second-order shape of the correlation surface $\left(\mathrm{a}_{11}, \mathrm{a}_{20}\right.$, and $\left.\mathrm{a}_{02}\right)$ are not dependent on the components of the translation vector $\left\{\mathrm{x}_{0}\right.$, $\left.\mathrm{y}_{0}\right\}$. Therefore, this method would consist of the following four steps.

a. Compute $\mathrm{a}_{11}, \mathrm{a}_{20}$, and $\mathrm{a}_{02}$ by applying equations (22) $\sim(24)$ to autocorrelation data obtained immediately after a reference frame has been acquired.

b. After each subsequent paper-fiber image has been captured and loaded into the comparison frame, compute $\mathrm{a}_{10}$ and $\mathrm{a}_{01}$ by applying (20) and (21) to the data obtained by cross-correlation the comparison and 
reference frames.

c. Substitute these values of $\mathrm{a}_{10}$ and $\mathrm{a}_{01}$, and the values of $\mathrm{a}_{11}, \mathrm{a}_{20}$, and $\mathrm{a}_{02}$ computed from the autocorrelation data, into (15) and (16) to obtain the translation coordinates of the current image.

d. Repeat this process after each new reference frame has been acquired.

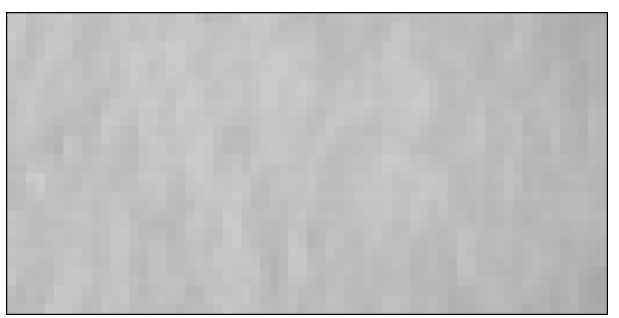

Fig. 9 Test image pattern

The performance is evaluated by the navigating vector error which is comparing with the real position and experiment position in correlation navigators $3 \times 3$ and $5 \times 5$ respectively using linear least-square method and another correlation surface. The navigation errors result from the correlation numbers which is obtained by each data coordinate using a linear, quadratic, cubic correlation function. The linear correlation function shows a large vector error in edge of correlation surface. Quadratic correlation function is more reduced vector error than linear method, but the error vector point in the pixel boundary presents as shown in Fig. 12. The cubic correlation function is reduced vector error than others. Fig. 10 and 11 show linear correlation method, Fig. 11 is vector error of center data block. The quadratic method shows in Fig. 12 and 13, Fig. 14 and 15 show the cubic method.

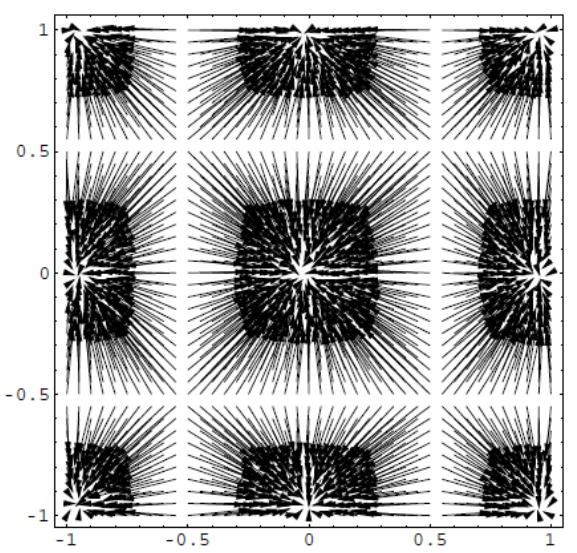

Fig. 10 Full data of navigation vector error by using linear correlation function.

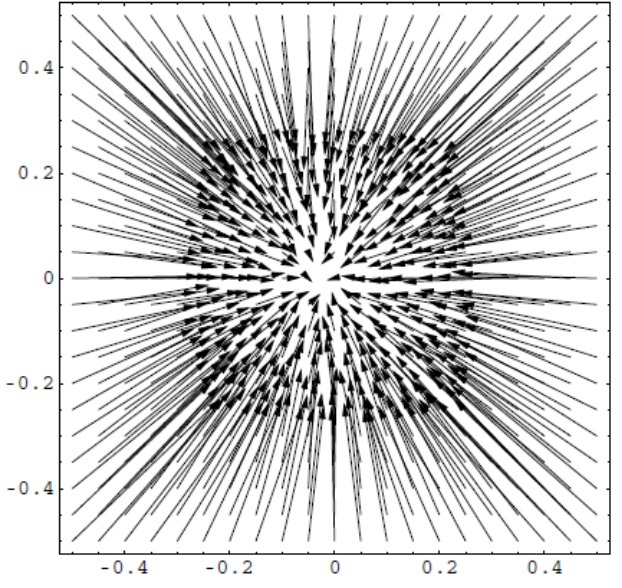

Fig. 11 Center data of navigation vector error by using linear correlation function.

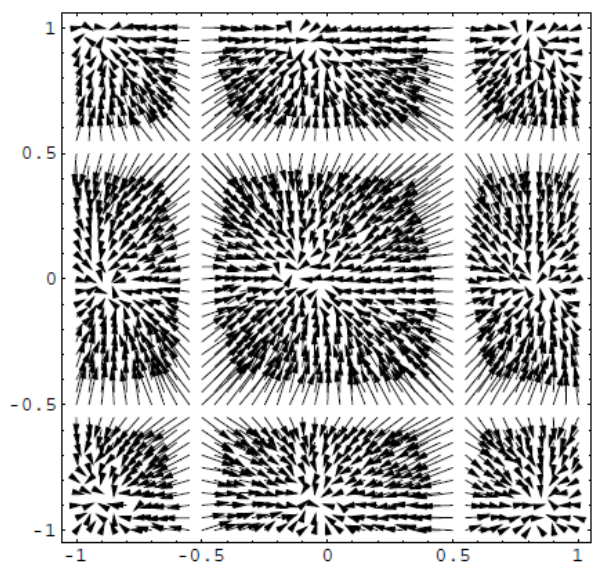

Fig. 12 Full data of navigation vector error by using quadratic correlation function

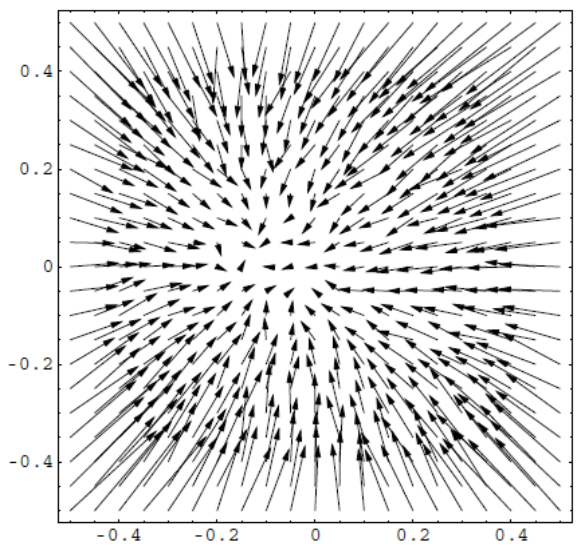

Fig. 13 Center data of navigation vector error by using quadratic correlation function 


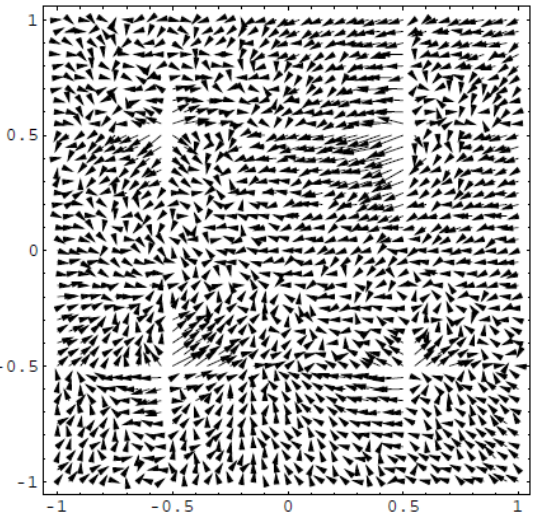

Fig. 14 Full data of navigation vector error by using cubic correlation function

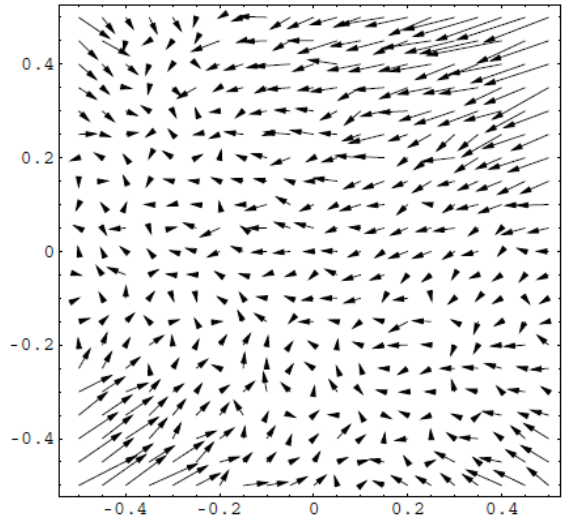

Fig. 15 Center data of navigation vector error by using cubic correlation function

The ideal vector error has 0.0 to navigate the coordinate on the one plane accurately. In the experimental results, the pixel error range is tolerated to max 0.39 and min 0.22 . The same as above processed, Fig. 13 shows the navigation errors of correlation numbers. The pixel error range is tolerated to 011 to 0.24 . The average comes out a shortage $23 \%$ to approach zero. Each error vector points to the real position known and the position computed by the algorithm. The closer to the origin point, the performance of the algorithm is quite good, whereas the navigation errors increase nonlinearly at distance from the origin point.

\section{CONCLUSIONS}

The algorithm is proposed to navigate the coordinate for stitching with separated CIS images sensing using correlation functions and to reduce navigation vector error in shifting the coordinate. The pixel correlation is detected in comparing to the reference image and a target image using the propose correlation function. The displacement of pixel in coordinate of two images can be obtained using the correlation surface function. For the accurate coordinate tracking, the cubic correlation function is introduced, and vector error is reduced. For the performance, the navigation path grid is expanded to grid number 25 with linear least-square method. The closer to the origin point is quite good, whereas the navigation errors increase nonlinearly at distance off the origin. The difference between the pixel position of the real image and the position vector in navigating makes sure the low error rate to be expanded the path navigating. In near future the vector error should be zero to navigate the vertical and horizontal coordinate, and enhance and restore a sensing image pattern.

\section{REFERENCES}

[1] Rafael C. Gonzalez and Richard E. Woods, Digital Image Processing, Prentice-Hall, 2002.

[2] Kwang Ryol Ryu, Young Bin Kim, Chang Woo Hur, Robert J.Sclabassi, "Moving Path Tracing of Image Central Position with Autocorrelation Function" KIMICS Spring Conference, pp.302305, Oct 2008

[3] James Davis, "Mosaics of Scenes with Moving Objects", in Proc. Conf. Computer Vision and Pattern Recognition, pp.354-360, 1998.

[4] Assaf Zomet, Anat Levin, Shmuel Peleg, Yair Weiss, "Seamless Image Stitching by Minimizing False Edges", Transactions on Image Processing, Vol.15, No:4, pp.969-977, Apr 2006.

[5] Richard Szeliski, "Image Alignment and stitching: A Tutorial", Microsoft Research, September 27. 2004

[6] Matthew Alun Brown, "Multi-Image Matching using Invariant Features", University of British Columbia, July 26. 2005.

[7] Jong-Woong Choe, Tae-Kyun Kim "A Study for Enhancement of Fluid Flow Image Strained By CCD's Characteristic", Korea Informatin Science Society, Vol.23, No.2, pp.387-390, Oct 1996.

[8] Ma B, Zimmermann T., Rohde M., Sinkelbach S., He F., Lindenmaier W., Dittmar K.E., "Use of Autostitch for automatic sitching of microscope images", Micron, Vol.38, No.5, pp.492-499, Jul 2007.

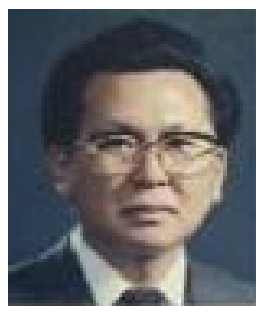

Kwang Ryol Ryu, PhD.

Received the $\mathrm{PhD}$. Degree from Kyunghee University in 1988, and is currently a professor at Dept. of Electronic Engineering at Mokwon University, worked as a visiting Professor at Dept. of Neurological Surgery at University of Pittsburgh Medical Center in USA in 2006 2008. His areas of research include Digital Signal Processing, Imaging and Vision, and Bio-Medical Electronics.

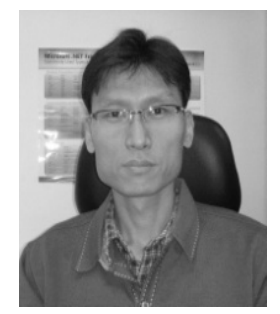

Young Bin Kim.

has been Ph.D. student at Mokwon University. He received his under graduate education and M.E. in Electronics Engineering from the Mokwon University in Korea. His areas of research include Digital Image Processing and DSP. 